\section{On the Interpretation of Shadow-moiré Fringes}

\section{Correction}

\section{by Authors}

Professor F. de Lamotte, of the Institute for Mathematics of the University of Liège, in Belgium has called to our attention several errors that regretfully had not been detected in our paper "On the Interpretation of Shadowmoiré Fringes". These errors have no effect on the approach followed nor on the conclusions but should be corrected before the proposed equations are used. The authors are very grateful to Prof. Lamotte for his comments and for checking carefully the derivations.

In eqs (A.4) and (A.5) the symbols $\tan \gamma$ and $\tan \phi$ should be exchanged. The equation therefore should read:

$$
e=\left(w+c+x \tan \theta_{1}\right) \tan \phi \sin \theta_{1} \tan \left(\theta_{1}-\phi\right)
$$

and

$$
g=\left(w+c+x \tan \theta_{1}\right) \tan \gamma \sin \theta_{1} \tan \left(\theta_{1}+\gamma\right)
$$

Also in the last term of eq (A.6) the values of $\tan \gamma$ and $\tan \phi$ should be interchanged and, consequently, in the general equation (A.13) there is a change in the factor of $\sin \theta_{1}$ which is carried to eq (1). These equations should read:

$$
\begin{aligned}
& a^{\prime}=\left(w+c+x \tan \theta_{1}\right)\left\{(\tan \gamma+\tan \phi) \cos \theta_{1}\right. \\
& \left.+\sin \theta_{1}\left[\tan \phi \tan \left(\theta_{1}-\phi\right)+\tan \gamma \tan \left(\gamma+\theta_{1}\right)\right]\right\}
\end{aligned}
$$

$$
\begin{gathered}
n=\frac{\left(w+c+x \tan \theta_{1}\right)}{p / \cos \theta_{3}}\left\{\left[\frac{\left[d-(h-z) \sin \theta_{2}+d_{1}\right] \tan \alpha-x}{d-(h-z) \sin \theta_{2}+d_{1}+w+c}\right.\right. \\
\left.\quad+\frac{x}{d-(h-z) \sin \theta_{2}+w+c}\right] \cos \theta_{1} \\
+\sin \theta_{1}\left[\frac { x } { d - ( h - z ) \operatorname { s i n } \theta _ { 2 } + w + c } \operatorname { t a n } \left(\theta_{1}-\tan ^{-1}\right.\right. \\
+\frac{\left[d-(h-z) \sin \theta_{2}+d_{1}\right] \tan \alpha-x}{d-(h-z) \sin \theta_{2}+d_{1}+w+c} \tan \left(\tan ^{-1}\right. \\
\quad \\
\left.\left.\left.\quad \frac{\left[d-(h-z) \sin \theta_{2}+d_{1}\right] \tan \alpha-x}{d-(h-z) \sin \theta_{2}+d_{1}+w+c}+\theta_{1}\right)\right]\right\}
\end{gathered}
$$

Only the results for case (a) are influenced by this correction and are governed by:

$n=\frac{1}{p}\left(w+c+x \tan \theta_{1}\right)\left[\tan \alpha \tan \theta_{1}+\sin \theta_{1} \tan \alpha \tan \left(\alpha+\theta_{1}\right)\right]$

and the sensitivity will be
Paper by J. Buitrago and A.J. Durelli was published in EXPERIMENTAL MECHANICS, June 1978, Pages 221-226

$$
s_{z}=\frac{1}{p} \sin \alpha \sec \left(\alpha+\theta_{1}\right)
$$

For case (b): $\theta_{1}=\theta_{3}=0$

and eq (4) should read:

$$
s_{x}=\frac{1}{p} \frac{f}{d-(h-z) \sin \theta_{2}+d_{1}}
$$

Because of typographical errors, eqs (8), (12) and (13) should be corrected as follows

$$
\begin{gathered}
w=-\frac{1}{2} k \pm \sqrt{\frac{1}{4} k^{2}+\frac{\left(d+\bar{d}_{1}\right) n p d}{\left(d+d_{1}\right) \tan \alpha-n p}} \\
k_{1}=\frac{d \tan \alpha+x-n p}{\tan \alpha} \\
n_{1,2}=\frac{w}{p} \tan \alpha
\end{gathered}
$$

and eqs (A.2) and (A.11) should read:

$$
\begin{aligned}
a^{\prime} & =\left[\left(a_{1}+a_{2}\right) \cos \theta_{1}+e+g\right] \\
\tan \gamma & =\frac{\left[d-(h-z) \sin \theta_{2}+d_{1}\right] \tan \alpha-x}{d-(h-z) \sin \theta_{2}+d_{1}+w+c}
\end{aligned}
$$

The variation of sensitivity with rotation $\theta_{1}$, about the $z$-axis for several orientations of the camera relative to the light source are shown in accompanying Fig. 1. It can be seen that, for small values of $\theta$ and $\alpha$, say less than 10 $\mathrm{deg}$, the fringe order is insensitive to the rotation $\theta_{1}$.

Fig. 1-Variation of sensitivity to the rotation $\theta_{1}$ about the $z$ axis for various relative inclinations $\alpha$ between camera axis and incident ray

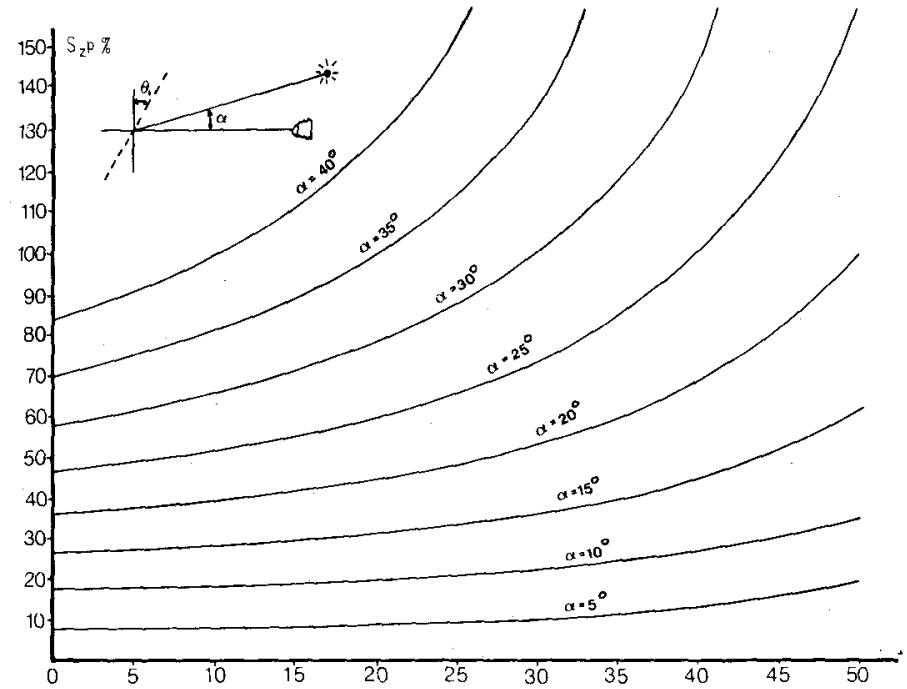

